ARTICLE

\title{
Giant tunnelling electroresistance in metal/ ferroelectric/semiconductor tunnel junctions by engineering the Schottky barrier
}

Zhongnan $\mathrm{Xi}^{1}$, Jieji Ruan ${ }^{2}$, Chen $\mathrm{Li}^{2}$, Chunyan Zheng ${ }^{1}$, Zheng Wen ${ }^{1,2,3}$, Jiyan Dai ${ }^{3}$, Aidong $\mathrm{Li}^{2} \& \mathrm{Di} \mathrm{Wu}^{2}$

Recently, ferroelectric tunnel junctions have attracted much attention due to their potential applications in non-destructive readout non-volatile memories. Using a semiconductor electrode has been proven effective to enhance the tunnelling electroresistance in ferroelectric tunnel junctions. Here we report a systematic investigation on electroresistance of $\mathrm{Pt} / \mathrm{BaTiO}_{3} / \mathrm{Nb}: \mathrm{SrTiO}_{3}$ metal/ferroelectric/semiconductor tunnel junctions by engineering the Schottky barrier on $\mathrm{Nb}: \mathrm{SrTiO}_{3}$ surface via varying $\mathrm{BaTiO}_{3}$ thickness and $\mathrm{Nb}$ doping concentration. The optimum ON/OFF ratio as great as $6.0 \times 10^{6}$, comparable to that of commercial Flash memories, is achieved in a device with $0.1 \mathrm{wt} \% \mathrm{Nb}$ concentration and a 4-unit-cell-thick $\mathrm{BaTiO}_{3}$ barrier. With this thinnest $\mathrm{BaTiO}_{3}$ barrier, which shows a negligible resistance to the tunnelling current but is still ferroelectric, the device is reduced to a polarization-modulated metal/semiconductor Schottky junction that exhibits a more efficient control on the tunnelling resistance to produce the giant electroresistance observed. These results may facilitate the design of high performance non-volatile resistive memories.

\footnotetext{
${ }^{1}$ College of Physics, Qingdao University, Qingdao 266071, China. ${ }^{2}$ National Laboratory of Solid State Microstructures, Department of Materials Science and Engineering, and Collaborative Innovation Center for Advanced Materials, Nanjing University, Nanjing 210093, China. ${ }^{3}$ Department of Applied Physics, The Hong Kong Polytechnic University, Hong Kong SAR 999077, China. Correspondence and requests for materials should be addressed to Z.W. (email: zwen@qdu.edu.cn) or to D.W. (email: diwu@nju.edu.cn).
} 
T he electrically switchable spontaneous polarization makes ferroelectric thin films promising candidate materials for non-volatile memories ${ }^{1,2}$. Ferroelectric random access memories have already been commercially available, which have the advantages of fast write speed and low power consumption ${ }^{1,2}$. However, the ferroelectric random access memories are based on capacitor structures and the readout is destructive, preventing the miniaturization of the devices, increasing the readout time and limiting the device lifetime $e^{1,2}$.

Recently, ferroelectric polarization switching has been achieved in ultrathin films only a few unit cells (u.c.) in thickness ${ }^{3-7}$. Resistive-type non-volatile ferroelectric memories, enabling high density integration and non-destructive readout, have been realized based on ferroelectric tunnel junctions (FTJs) that are composed of two metal electrodes separated by an ultrathin ferroelectric as the barrier ${ }^{8-15}$. The overall effective barrier of an FTJ can be modulated by the polarization reversal in the ferroelectric barrier, giving rise to non-volatile switching of the tunnelling resistance between a high (OFF state) and a low (ON state) value, that is, the so-called tunnelling electroresistance $(\text { TER })^{8-10}$. The TER characteristic has been demonstrated in $\mathrm{BaTiO}_{3}$ (BTO) ultrathin films using metallic $\left(\mathrm{La}_{0.67} \mathrm{Sr}_{0.33}\right) \mathrm{MnO}_{3}$ or $\mathrm{SrRuO}_{3}$ as one electrode and a conductive tip in a scanning probe microscope as the other electrode ${ }^{11,12}$. Later, Chanthbouala et al. ${ }^{13}$ reported a high speed write/read with nanosecond pulses and a power consumption as low as femto-joule per bit in $\mathrm{Au} / \mathrm{Co} / \mathrm{BTO} /$ $\left(\mathrm{La}_{0.67} \mathrm{Sr}_{0.33}\right) \mathrm{MnO}_{3}$ FTJs. The TER behaviour has also been observed in a number of other metal/ferroelectric/metal tunnel junctions, such as $\mathrm{Co} / \mathrm{Pb}(\mathrm{Zr}, \mathrm{Ti}) \mathrm{O}_{3} /\left(\mathrm{La}_{0.70} \mathrm{Sr}_{0.30}\right) \mathrm{MnO}_{3}$ (ref. 14), $\mathrm{Pt} / \mathrm{BTO} / \mathrm{SrRuO}_{3}$ (ref. 16), $\mathrm{Cr} / \mathrm{BTO} / \mathrm{Pt}$ (ref. 17), $\left(\mathrm{La}_{0.67} \mathrm{Sr}_{0.33}\right) \mathrm{MnO}_{3} /$ $\mathrm{BiFeO}_{3} /\left(\mathrm{La}_{0.67} \mathrm{Sr}_{0.33}\right) \mathrm{MnO}_{3}$ (ref. 18) and $\mathrm{Co} / \mathrm{BiFeO}_{3} / \mathrm{LaNiO}_{3}$ (ref. 19), in which the ON/OFF current ratio originates from a modulation on the height of the ferroelectric barrier. Recently, efforts have been made to enhance the TER performance by incorporating extra barriers between the ferroelectric barrier and the metallic electrode, for example, the $\mathrm{CoO}_{\mathrm{x}}$ layer at $\mathrm{Co} / \mathrm{BTO}$ interface ${ }^{20}$, the ultrathin dielectric $\mathrm{SrTiO}_{3}$ barrier at $\mathrm{BTO} / \mathrm{SrRuO}_{3}$ interface ${ }^{21,22}$ and the metal-insulator phase transition insertion $\left(\mathrm{La}_{0.50} \mathrm{Ca}_{0.50}\right) \mathrm{MnO}_{3}$ at $\mathrm{BTO} /\left(\mathrm{La}_{0.70} \mathrm{Sr}_{0.30}\right) \mathrm{MnO}_{3}$ interface ${ }^{23}$. In these FTJs, the extra barriers vary in height and/or in width in response to the polarization reversal and give a more efficient modulation on the junction transport.

Previously, we have proposed a metal/ferroelectric/semiconductor (MFS)-type FTJ. In this device, there is an extra Schottky barrier on the depleted semiconductor surface, which varies both in width and in height in response to the ferroelectric polarization reversal due to a ferroelectric field effect. The non-volatile modulation on both the ferroelectric barrier and the extra Schottky barrier gives rise to a greatly enhanced ON/OFF ratio ${ }^{24}$. Using $\mathrm{Nb}: \mathrm{SrTiO}_{3}$ (NbSTO) as the semiconductor electrode, an ON/OFF ratio of $10^{4}$ has been achieved in a $\mathrm{Pt} / \mathrm{BTO} / \mathrm{NbSTO}$ device ${ }^{24}$. More recently, Hu et al. ${ }^{25}$ have reported a variation of electroresistance by about ten times in $\mathrm{ITO} / \mathrm{Pt} / \mathrm{BiFeO}_{3} / \mathrm{NbSTO}$ FTJs under UV illumination, due to an optical modulation on the Schottky barrier in NbSTO. These results demonstrate the significance of the interface Schottky barrier on the performance of MFS tunnel junctions.

Here we show that, by engineering the Schottky barrier, a giant ON/OFF ratio, about $6.0 \times 10^{6}$, can be achieved in an optimized $\mathrm{Pt} / \mathrm{BTO} / \mathrm{NbSTO}$ FTJ with a 4 u.c.-thick BTO barrier and a $\mathrm{Nb}$ concentration of $0.1 \mathrm{wt} \%$. In particular, the 4 u.c.-thick BTO barrier provides a switchable polarization but shows a negligible resistance to electron tunnelling, as compared with the contribution from the Schottky barrier. Therefore, the TER comes completely from a polarization-modulation on the metal/semiconductor interface Schottky barrier. This is different from previously reported MFS-type FTJs ${ }^{24}$, where the interface Schottky barrier works together with the ferroelectric barrier. This polarization-modulation produces a more efficient control on the tunnelling transport through the device and generates the giant $\mathrm{ON} / \mathrm{OFF}$ ratio comparable to commercial Flash memories ${ }^{26-29}$.

\section{Results}

Ferroelectric properties of ultrathin BTO films. Piezoresponse force microscopy (PFM) is used to characterize ferroelectric properties of the ultrathin BTO films. As shown in Fig. 1a, the PFM signal is collected with a conductive tip in contact with the film surface, as the NbSTO electrode is grounded. The step-terrace surface morphology with a step height of $0.4 \mathrm{~nm}$, measured by atomic force microscopy (AFM), indicates a layer-by-layer growth, which is also demonstrated by the intensity oscillation in reflection high energy electron diffraction collected in situ during the deposition (Supplementary Fig. 1). PFM hysteresis loops are measured from bare BTO surface using the waveform shown schematically in the inset in Fig. 1a, where a pulse sequence following a triangle profile is used to switch the film and the remnant piezoresponse is measured by a small-signal AC voltage $\left(V_{\mathrm{AC}}\right)$ applied following each pulse $^{30}$. The remnant piezoresponse hysteresis loop (see Methods section) of a 4 u.c.-thick BTO measured with $V_{\mathrm{AC}}=1.0 \mathrm{~V}$ is shown in Fig. 1b. However, the PFM measurement is sensitive to the contact between the tip and the sample surface and similar hysteresis loops have also been observed in non-ferroelectric materials, such as $\mathrm{LaAlO}_{3} / \mathrm{SrTiO}_{3}$ heterostructures and amorphous $\mathrm{HfO}_{2}$ thin films, owing to charge injection, ionic motion and interfacial electrochemical reaction ${ }^{31,32}$. To distinguish the piezoresponse hysteresis due to ferroelectric polarization reversal, Strelcov et al. ${ }^{33}$ proposed a $V_{\mathrm{AC}}$-dependent PFM measurement, in which the remnant piezoresponse hysteresis loop of a ferroelectric material deforms and even collapses when $V_{\mathrm{AC}}$ is above the coercive voltage because the polarization flipping during measurement cancels the piezoresponse. However, this reduction of the measured piezoresponse above a critical $V_{\mathrm{AC}}$ value is absent in non-ferroelectrics ${ }^{31,32}$. The piezoresponse loops as a function of $V_{\mathrm{AC}}$ are shown in Fig. $1 \mathrm{~b}$. The hysteresis starts to reduce at $V_{\mathrm{AC}}=3.2 \mathrm{~V}$, just above the coercive voltage, and then reduces abruptly as $V_{\mathrm{AC}}$ further increases, in good agreement with the results reported previously in $\mathrm{Pb}(\mathrm{Zr}, \mathrm{Ti}) \mathrm{O}_{3}$ and $\mathrm{BiFeO}_{3}$ thin films ${ }^{31,32}$. The PFM amplitude and phase loops measured at $V_{\mathrm{AC}}=3.2$ and $4.4 \mathrm{~V}$ are shown in the inset for clarity. Similar reduction in piezoresponse when $V_{\mathrm{AC}}$ is greater than the coercive voltage is also observed from $\mathrm{Pt}$ top electrode deposited on the BTO/NbSTO heterostructure (Supplementary Fig. 2a-c). These observations evidence robust ferroelectricity of the 4 u.c.-thick BTO film deposited on NbSTO. Figure 1d,e shows PFM out-of-plane phase and amplitude images of domains on this BTO film patterned according to the protocol shown in Fig. 1c. The $180^{\circ}$ phase contrast and the clear domain boundary indicate that antiparallel domains can be written in this ultrathin BTO layer.

The effects of ferroelectric barrier thickness. The FTJ devices are defined by depositing Pt top electrodes on BTO/NbSTO heterostructures. Figure 2a depicts band alignment of separated $\mathrm{Pt}, \mathrm{BTO}$ and NbSTO, assuming for clarity that the BTO is not polarized. The contact potential, arising from the difference between the work function of Pt $\left(\Phi_{\mathrm{Pt}}=5.65 \mathrm{eV}\right)$ and the Fermi level $\left(E_{\mathrm{F}}\right)$ of NbSTO, is shared by the BTO barrier layer and the depleted region on the surface of NbSTO (Fig. 2b) ) $^{34,35}$ 
a

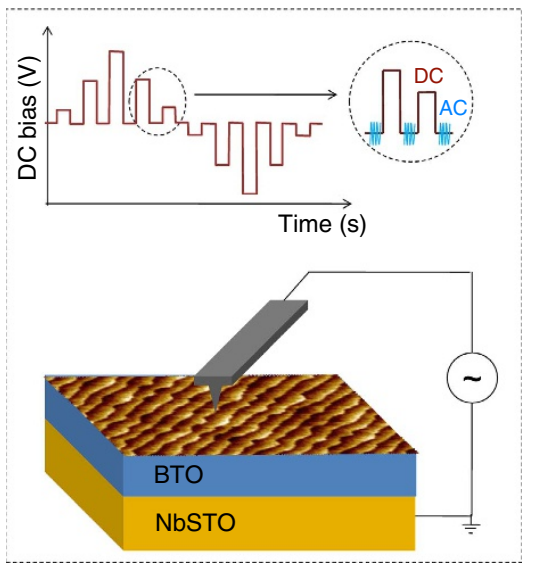

b
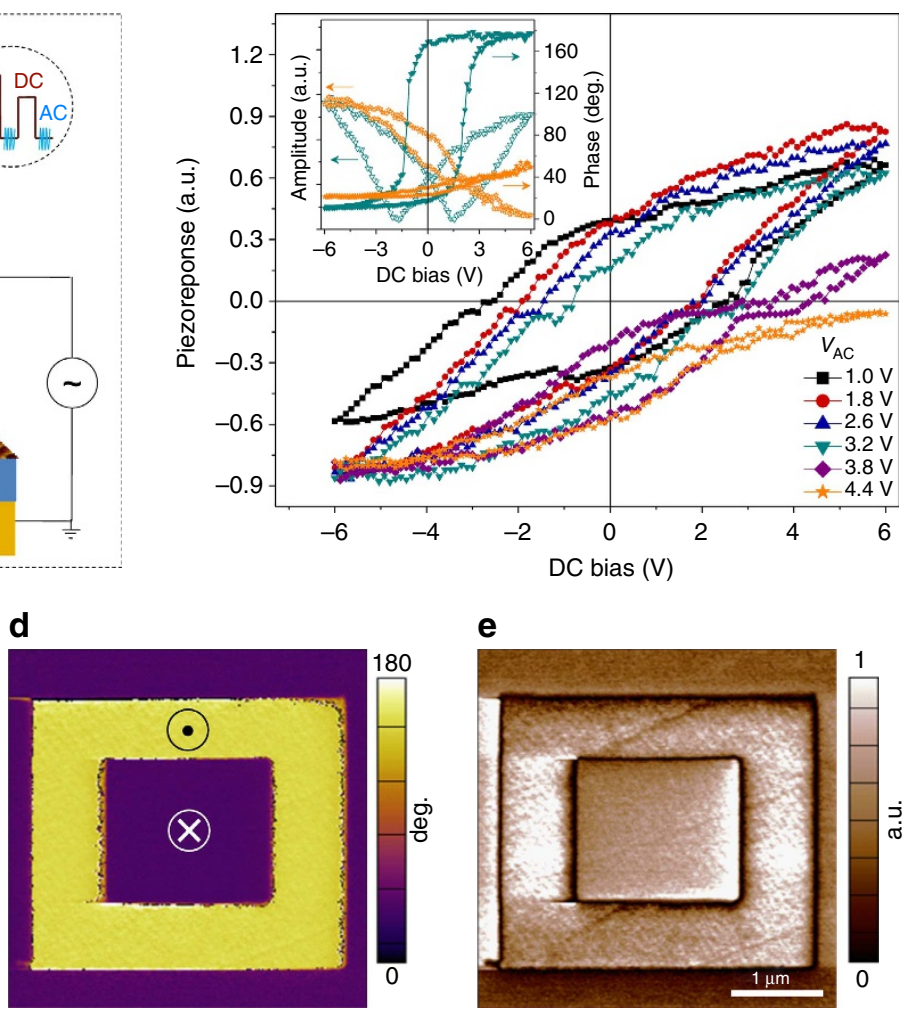

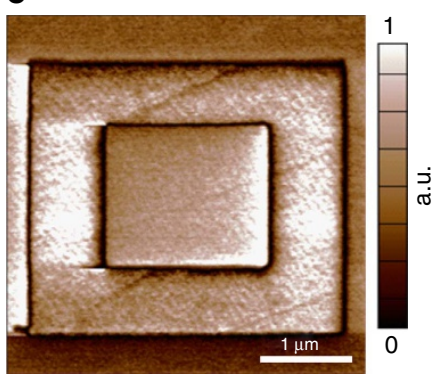

Figure 1 | Topography and ferroelectric properties of a 4 u.c.-thick $\mathrm{BaTiO}_{3}$ film deposited on $\mathbf{N b}: \mathrm{SrTiO}_{3}$ substrate. (a) Atomic force microscopy surface morphology and a schematic description of the piezoresponse force microscopy (PFM) measurements. The inset shows the applied pulse train for hysteresis measurements. (b) Remnant PFM piezoresponse hysteresis loops collected with various $V_{\text {AC }}$ values. The inset shows amplitude and phase hysteresis loops with $V_{\mathrm{AC}}=3.2 \mathrm{~V}$ (dark green) and $4.4 \mathrm{~V}$ (orange). (c) Protocol for domain patterning. (d) PFM phase and (e) PFM amplitude images acquired after the domain patterning.

A Schottky barrier appears even though the BTO is assumed unpolarized. With decreasing BTO thickness, the potential that drops on the BTO layer decreases and the Schottky barrier on the depleted semiconductor surface enhances accordingly, as shown in Fig. 2c. The evolution of the energy profile with decreasing BTO thickness is reminiscent of the formation of a metal/ semiconductor Schottky junction ${ }^{34}$. If the BTO thickness decreases to zero, the $\mathrm{Pt} / \mathrm{BTO} / \mathrm{NbSTO}$ structure reduces to a Pt/NbSTO Schottky contact. When the BTO barrier with a finite thickness is polarized, the ferroelectric bound charges at the semiconductor surface modulate the profile of the Schottky barrier via a ferroelectric field effect. This modulation results in the polarization-dependent transport through the junction, that is, the TER effect. This TER behaviour is confirmed by combined PFM and conductive AFM measurements on the Pt top electrode of a $\mathrm{Pt} / \mathrm{BTO} / \mathrm{NbSTO}$ device, as shown in Supplementary Fig. $2 \mathrm{~d}-\mathrm{f}$. With the polarization pointing to NbSTO, the positive bound charges tend to suppress the Schottky barrier since the depleted region on the semiconductor surface is reduced or even annihilated by electron accumulation. The device is in the ON state with a larger current. As the polarization is switched pointing to $\mathrm{Pt}$, the negative bound charges on the semiconductor surface help to sweep the electrons away from the ferroelectric/semiconductor interface and enhances the Schottky barrier. The junction current is suppressed associated with the appearance of a typical rectifying character. The device is then switched to the OFF state.

Room-temperature resistance-voltage hysteresis loops of the $\mathrm{Pt} / \mathrm{BTO} / \mathrm{NbSTO}(\mathrm{Nb}: 0.7 \mathrm{wt} \%$ ) FTJs as a function of BTO thickness are shown in Supplementary Fig. 3. The critical voltages for resistance switching between the ON and the OFF states coincide with the coercive voltages of the PFM hysteresis loops, which again evidences the correlation between resistive switching and the polarization reversal. Room-temperature current-voltage $(I-V)$ curves for the $\mathrm{ON}$ and the OFF states of the $\mathrm{Pt} / \mathrm{BTO} /$ NbSTO (Nb: $0.7 \mathrm{wt} \%)$ FTJs as a function of BTO thickness are shown in Fig. 2d,e, respectively. The write pulse height was determined from the resistance-voltage hysteresis loop for each device (Supplementary Fig. 3). Compared with the ON state transport characteristics in Fig. 2d, the OFF state $I-V$ curves in Fig. 2e show a more pronounced variation with decreasing BTO thickness. Figure $2 \mathrm{f}$ shows the ON and the OFF current, as well as the ON/OFF ratio, as a function of BTO thickness. It is clear that the ON state current exhibits only a slight variation, while the OFF state current decreases about three orders of magnitude with decreasing BTO thickness from 16 down to 4 u.c., resulting in a dramatic increase in ON/OFF ratio from about 400 to $8 \times 10^{5}$. The suppression of the OFF state current with decreasing BTO thickness is associated with the appearance of a rectifying transport character (Fig. 2e). This indicates that the Schottky barrier becomes more prominent in FTJs with a thinner BTO barrier, as shown in Fig. 2a-c. In fact, it is observed that the $I-V$ characteristic of an In/BTO/ NbSTO device with the thinnest BTO barrier is almost identical to an In/NbSTO Ohmic contact (Supplementary Fig. 4), indicating that the 4 u.c.-thick ultrathin BTO layer exhibits a negligible resistance to the tunnelling current. The $\mathrm{Pt} / \mathrm{BTO} / \mathrm{NbSTO}$ FTJ with a 4 u.c.-thick BTO barrier is then 
a

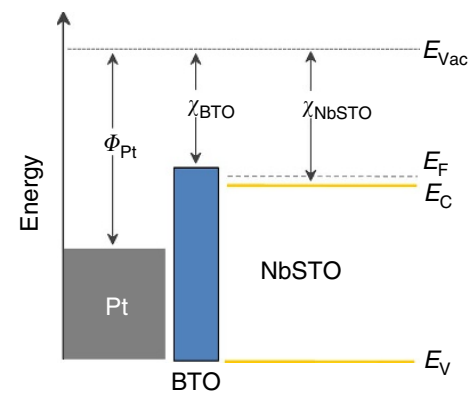

d

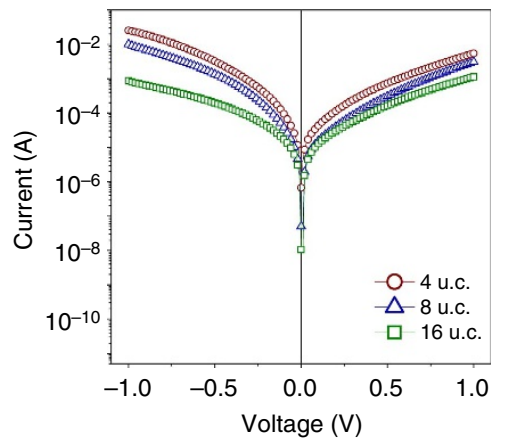

b

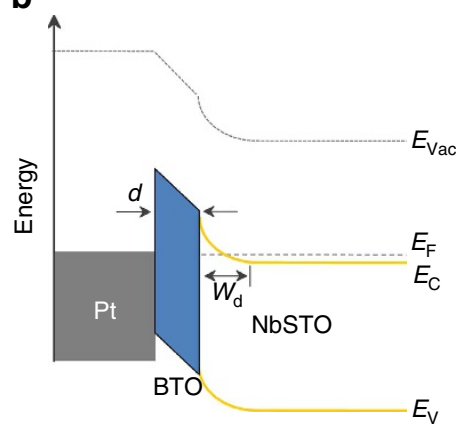

C

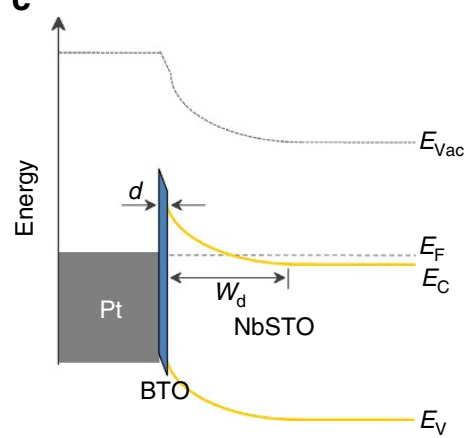

e

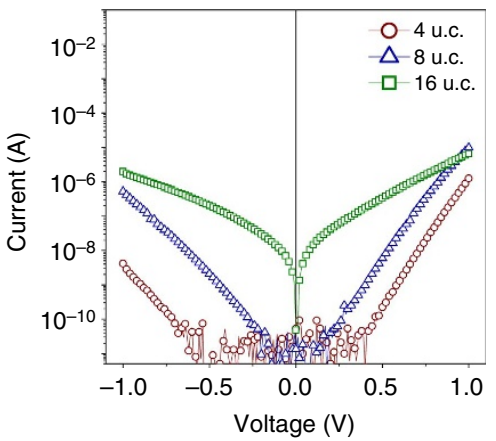

f

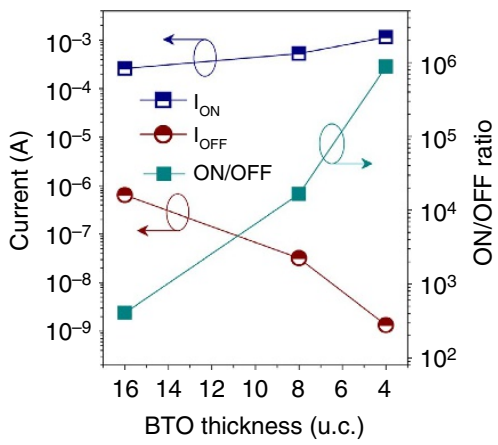

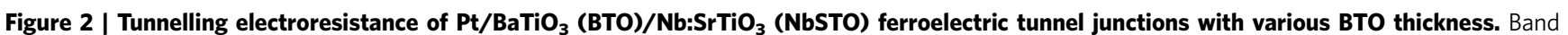
diagram of separated Pt, BTO and NbSTO (a) and of Pt/BTO/NbSTO junction with a thick (for example, 16 u.c.) (b) and a thin (for example, 4 u.c.) BTO barrier (c), where $\Phi_{\mathrm{Pt}}=5.65 \mathrm{eV}$ is the work function of Pt, $\chi_{\mathrm{B} T \mathrm{O}}=3.9 \mathrm{eV}$ the electron affinity of BTO, $\chi_{\mathrm{NbSTO}}=4.08 \mathrm{eV}$ the electron affinity of NbSTO, $E_{\mathrm{Vac}}$ the vacuum level, $E_{\mathrm{C}}, E_{\mathrm{V}}$ and $E_{\mathrm{F}}$ the conduction band minimum, the valence band maximum and the Fermi level of NbSTO, respectively, $d$ the BTO thickness, $W_{d}$ the depletion region width. (d,e) I-V curves of Pt/BTO/NbSTO (Nb: 0.7 wt\%) FTJs, with a BTO thickness of 4, 8 and 16 u.c., in the ON and the OFF states, respectively. (f) Junction currents and corresponding ON/OFF ratios, read at $0.6 \mathrm{~V}$, as a function of BTO thickness.
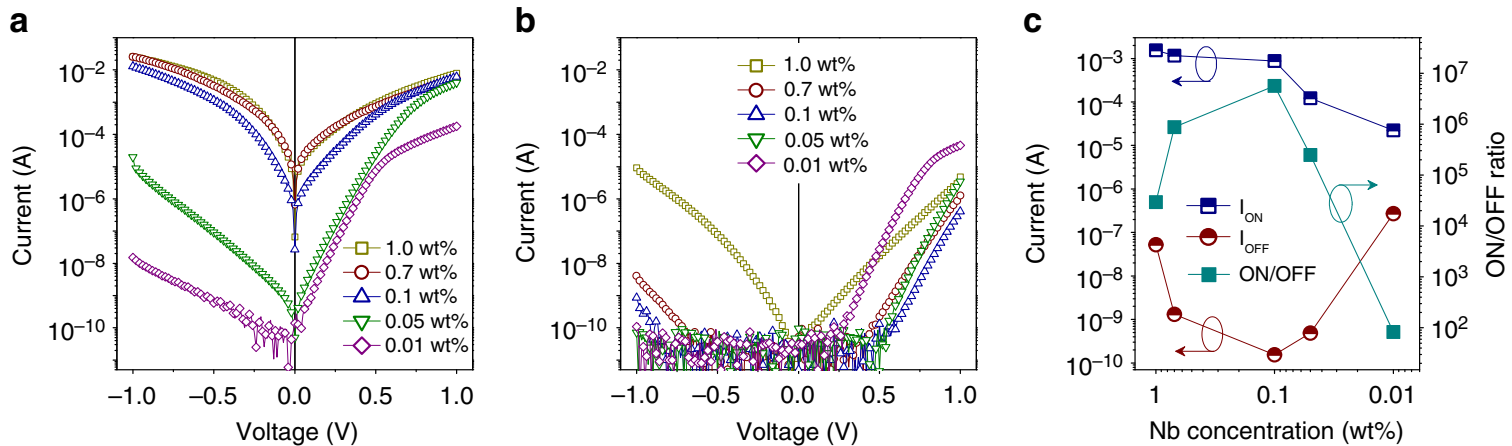

Figure 3 | Tunnelling electroresistance of $\mathrm{Pt}^{-\mathrm{BaTiO}_{3}}$ (BTO)/Nb:SrTiO ${ }_{3}$ ferroelectric tunnel junctions with a 4 u.c.-thick $\mathrm{BTO}$ barrier as a function of $\mathbf{N b}$ concentration. $(\mathbf{a}, \mathbf{b}) I-V$ curves for the ON and the OFF state, respectively. (c) Junction currents and ON/OFF ratios, read at $0.6 \mathrm{~V}$.

reduced to a polarization-modulated $\mathrm{Pt} / \mathrm{NbSTO}$ metal/semiconductor Schottky junction. Figure 2f shows that such a modulation on the Schottky junction generates an improved ON/OFF ratio, as a result of the efficient shut-off of the OFF state current due to the enhanced Schottky barrier.

The effects of Nb doping concentration. The TER characteristics of the polarization-modulated metal/semiconductor Schottky junction can be further improved by optimizing the $\mathrm{Nb}$ doping concentration in NbSTO. I-V curves of the $\mathrm{ON}$ and the OFF states for Pt/BTO/NbSTO FTJs with a 4 u.c.-thick BTO barrier and various $\mathrm{Nb}$ concentration from 0.01 to $1.0 \mathrm{wt} \%$ are shown in Fig. 3a,b, respectively. The ON state $I-V$ curves at the
$\mathrm{Nb}$ concentrations of 1.0 and $0.7 \mathrm{wt} \%$ are relatively symmetric for the forward and the reverse bias. However, it becomes more and more asymmetric with decreasing $\mathrm{Nb}$ concentration. The OFF state current shows a clear rectifying character, except for the junction with the highest $\mathrm{Nb}$ concentration of $1.0 \mathrm{wt} \%$. The junction currents and the corresponding ON/OFF ratios are plotted in Fig. 3c, as a function of $\mathrm{Nb}$ concentration. As shown, the ON/OFF ratio increases from $3 \times 10^{4}$ to $6 \times 10^{6}$ with decreasing $\mathrm{Nb}$ concentration from 1.0 to $0.1 \mathrm{wt} \%$, owing to the dramatic decrease of the OFF state current. The suppression of the OFF state current is accompanied with the increase in the onset voltage of the forward current (Fig. 3b). However, as the $\mathrm{Nb}$ concentration further decreases from 0.1 to $0.01 \mathrm{wt} \%$, the ON/OFF ratio decreases abruptly. It reduces to only about 80 at 

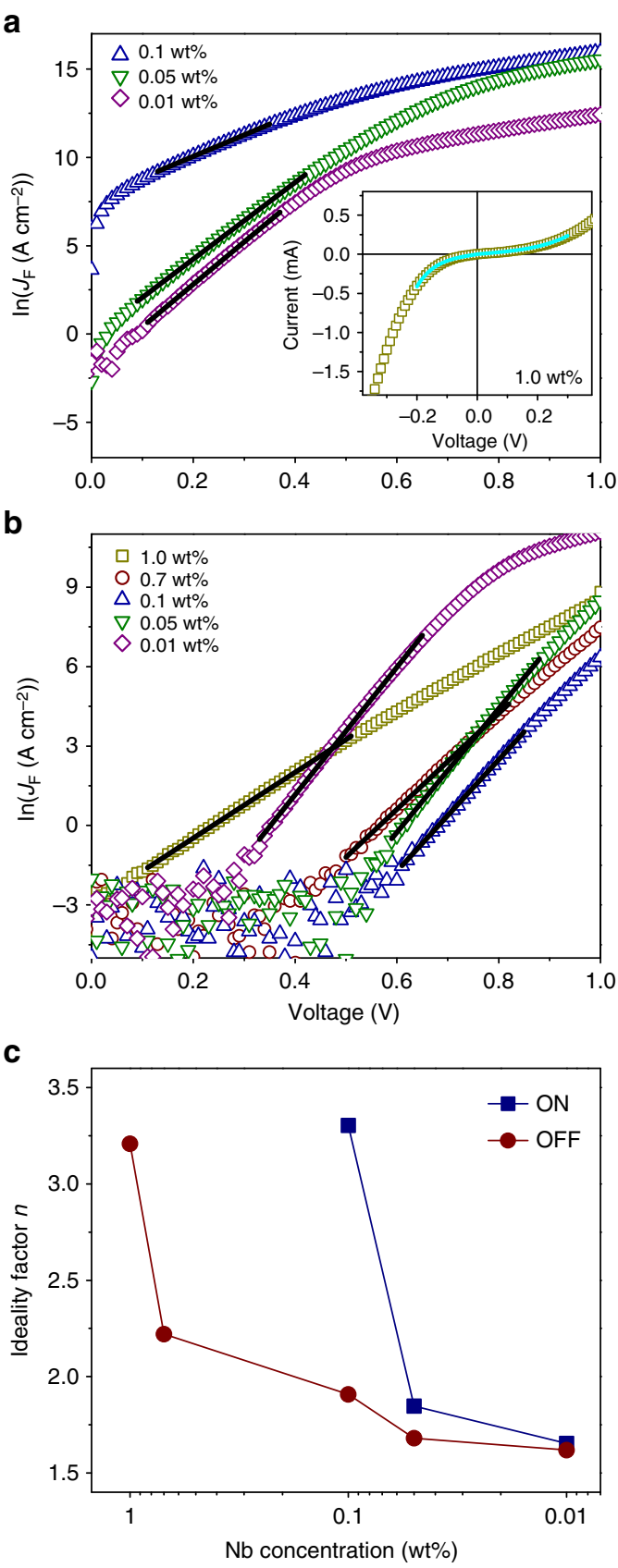

Figure 4 | Fits to the I-V curves of $\mathrm{Pt} / \mathrm{BaTiO}_{3}(\mathrm{BTO}) / \mathrm{Nb}: \mathrm{SrTiO}_{3}$ ferroelectric tunnel junctions with a 4 u.c.-thick BTO barrier. $(\mathbf{a}, \mathbf{b}) \ln J_{\mathrm{F}}-V$ plots for the ON and the OFF states, respectively. The black solid lines are fits to equation (1). The inset in $\mathbf{a}$ is the $\mathrm{ON}$ state $I-V$ curve of the junction with a $\mathrm{Nb}$ concentration of $1.0 \mathrm{wt} \%$, where the cyan solid line is a fit to the direct tunnelling model. (c) Ideality factor $n$ extracted from the fitting as a function of $\mathrm{Nb}$ concentration.

the lowest $\mathrm{Nb}$ concentration of $0.01 \mathrm{wt} \%$, as shown in Fig. 3c. With the decrease of TER, the ON state current decreases by more than an order (Fig. 3a), whereas the OFF state current increases significantly by almost four orders. In addition, the increase of OFF state current is associated with the decrease in the onset voltage of the forward current (Fig. 3b).

To gain insight into the complicated TER characteristics, the $I-V$ curves are fitted to different transport models. In the heavily doped regime with $\mathrm{Nb}$ concentration at 1.0 and $0.7 \mathrm{wt} \%$, the ON state $I-V$ curves are identical and nonlinear. The low-voltage part of the $I-V$ curves can be well fitted to the direct tunnelling model based on a trapezoidal potential barrier (see Methods section) ${ }^{12}$. As shown in the inset in Fig. 4a, the validity of the direct tunnelling model implies that the Schottky barrier on the heavily doped NbSTO surface is eliminated when the BTO polarization points to the NbSTO in the ON state. The BTO barrier, extracted from the fitting, is 1.7 and $0.2 \mathrm{eV}$ in height for the $\mathrm{Pt} / \mathrm{BTO}$ and the BTO/NbSTO interfaces, respectively. The $I-V$ curve at higher voltage is dominated by Fowler-Nordheim tunnelling through a triangular barrier, as reported previously in metal/ferroelectric/metal $\mathrm{FTJ}^{10,36}$. As the $\mathrm{Nb}$ concentration decreases, the $\mathrm{ON}$ state currents at forward bias fit best to an exponential increase with the applied voltage at low voltage (Fig. 4a), suggesting the depletion of the NbSTO surface and the dominance of the Schottky barrier on the transport. However, the transport at higher voltage is dominated probably by a bulk-limited conduction mechanism ${ }^{37}$. On the other hand, as BTO polarization points to the Pt electrode in the OFF state, the Schottky barrier is enhanced due to the ferroelectric field effect and the $I-V$ curves, in all the FTJ devices, exhibit a thermally activated character. The junction transport controlled by a Schottky barrier is given by ${ }^{34}$

$$
J_{\mathrm{F}}=J_{0} \exp \left(q V / n k_{\mathrm{B}} T\right)
$$

where $J_{\mathrm{F}}$ is the forward current density, $J_{0}$ the saturated current density, $q$ the electron charge, $n$ the ideality factor, $k_{\mathrm{B}}$ the Boltzmann constant and $T$ the absolute temperature. As reported previously, $n$ equals unity if the transport is an ideal thermionic emission over a Schottky barrier ${ }^{34,38-42} . n$ extracted from the fits is plotted in Fig. 4c as a function of $\mathrm{Nb}$ concentration. It is clear that $n$ decreases as the device is switched from the $\mathrm{ON}$ to the OFF state and $n$ decreases from 3.4 to 1.6 with decreasing $\mathrm{Nb}$ concentration from 1.0 to $0.01 \mathrm{wt} \%$. The deviation of $n$ from unity may be ascribed to thermally assisted tunnelling as observed previously in NbSTO-based Schottky junctions ${ }^{34,38-40}$ and the presence of the ultrathin BTO layer, which shares the voltage applied on the junction.

In Pt/BTO/NbSTO FTJs, $n$ can be expressed as ${ }^{39,40}$,

$$
n=1+\frac{C_{\mathrm{d}}}{C_{\mathrm{f}}}=1+\frac{d}{W_{\mathrm{d}}} \cdot \frac{\varepsilon_{\mathrm{r}}}{\varepsilon_{\mathrm{f}}}
$$

where $C_{\mathrm{d}}$ and $C_{\mathrm{f}}$ are the high-frequency capacitances of the depleted region and the BTO barrier layer, $\varepsilon_{\mathrm{r}}$ and $\varepsilon_{\mathrm{f}}$ their respective relative dielectric constants, and $W_{\mathrm{d}}$ the depleted region width. Here, $\varepsilon_{\mathrm{r}}$ of NbSTO is assumed to be 290 at room temperature, following Barrett's formula (Supplementary Information $)^{41}$. According to equation (2), with a certain BTO thickness, the decrease of $n$ indicates the increase in $W_{\mathrm{d}}$. The build-in potential $\left(V_{\mathrm{bi}}\right)$ of a metal/semiconductor Schottky barrier is often analysed by capacitance-voltage measurements. However, in the present study with the presence of the ultrathin BTO layer, the depleted region capacitance $C_{\mathrm{d}}$ should be $n C$, where $C$ is the high-frequency capacitance of the whole junction, and the voltage that drops on the depleted region $\left(V_{\mathrm{d}}\right)$ should be calibrated as $V / n$, where $V$ is the voltage applied.

The room-temperature $C_{\mathrm{d}}^{-2}-V_{\mathrm{d}}$ characteristics of the $\mathrm{Pt} / \mathrm{BTO} / \mathrm{NbSTO}$ FTJs with a 4 u.c.-thick BTO barrier for the ON and the OFF states are shown in Fig. 5a,b, respectively, as a function of $\mathrm{Nb}$ concentration. The ON state capacitance for the device with the $\mathrm{Nb}$ concentration at $1.0 \mathrm{wt} \%$ is not shown because the Schottky barrier is completely annihilated by the electron accumulation. As shown, $C_{\mathrm{d}}^{-2}$ increases linearly with reverse bias, indicating that the permittivity of NbSTO is constant in the depleted region ${ }^{35,42,43}$. Similar linear $C_{\mathrm{d}}^{-2}-V_{\mathrm{d}}$ behaviours have also been observed in Au/NbSTO Schottky junctions, in which the $\varepsilon_{\mathrm{r}}$ of $\mathrm{NbSTO}$ exhibits a significant 
a

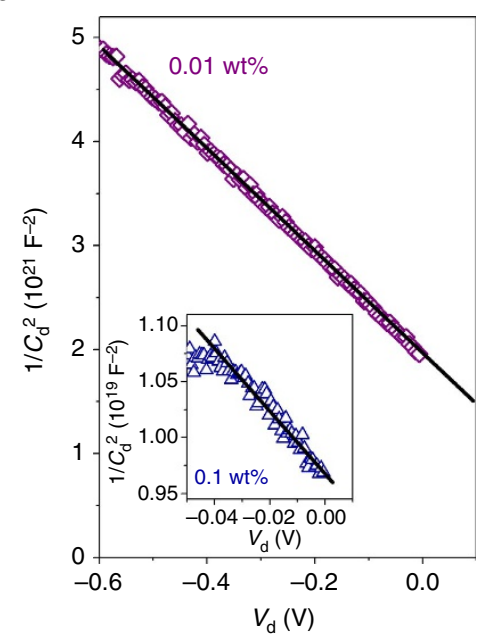

f

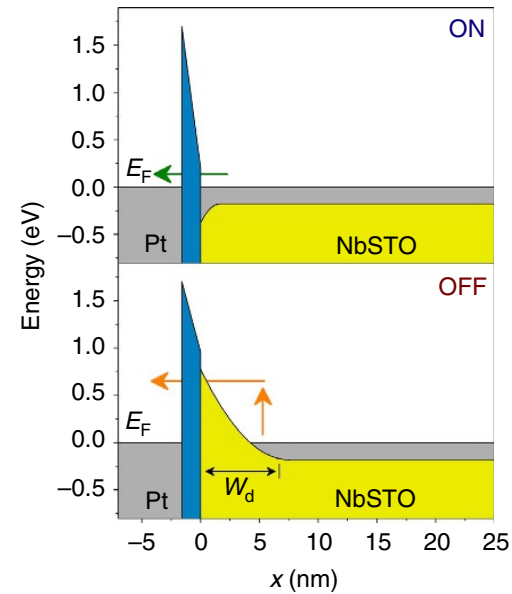

b

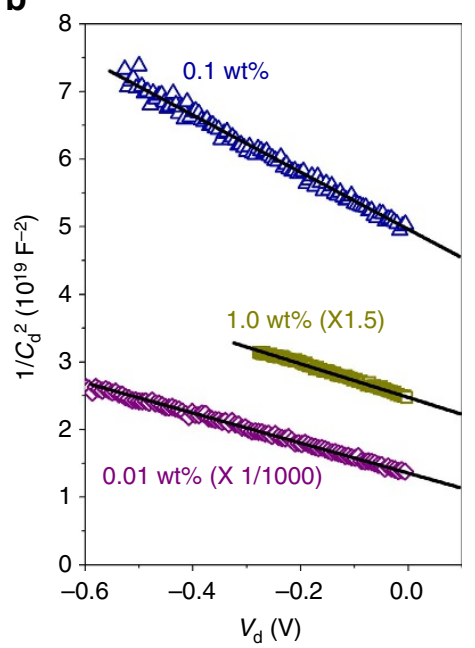

g

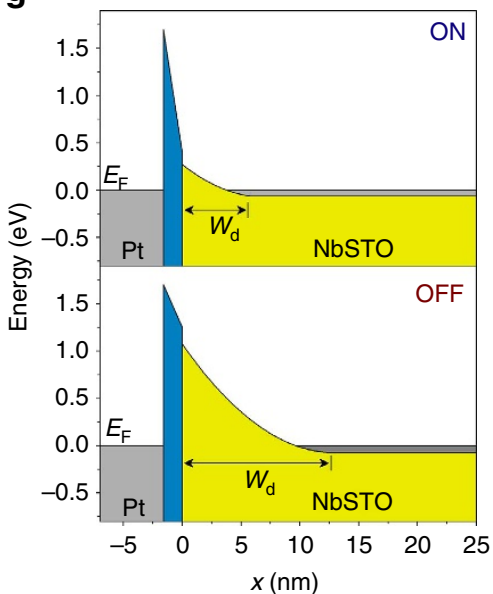

C

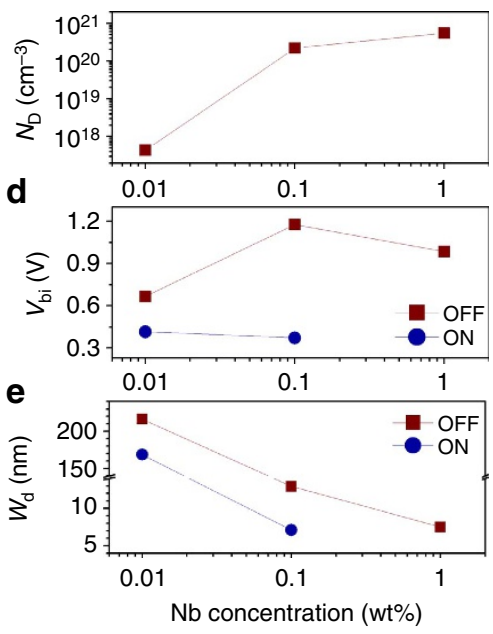

h

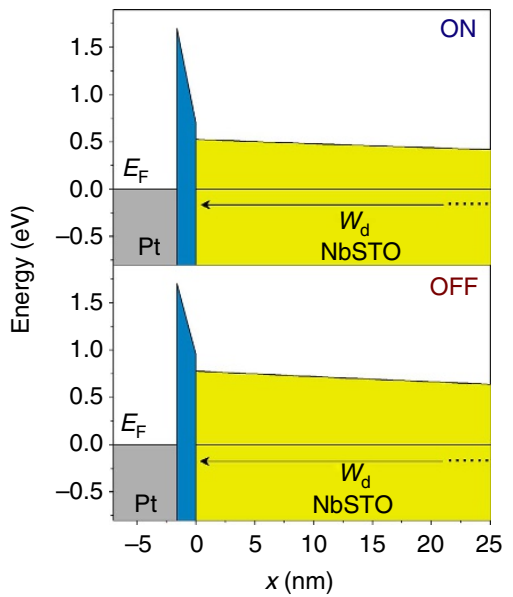

Figure 5 | C-V characteristics and energy profiles of $\mathrm{Pt} / \mathrm{BaTiO}_{3}$ (BTO)/Nb:SrTiO ${ }_{3}$ ferroelectric tunnel junctions with a 4 u.c.-thick BTO barrier and various $\mathbf{N b}$ concentration. $(\mathbf{a}, \mathbf{b}) C_{d}^{-2}-V_{d}$ plots for the $O N$ and the OFF states, respectively, where the black solid lines are fits to $C_{d}^{-2}=2\left(V_{b i}-V_{d}\right) / q \varepsilon_{0} \varepsilon_{r} N_{D} S^{2}$. The inset in $\mathbf{a}$ is the ON state $C_{d}^{-2}-V_{d}$ of the 0.1 wt\% device. (c) $N_{D}$ and (d) $V_{b i}$, extracted from the fits, as a function of $\mathrm{Nb}$ concentration. (e) $\mathrm{W}_{\mathrm{d}}$ of the $\mathrm{ON}$ and the OFF states as a function of $\mathrm{Nb}$ concentration. $(\mathbf{f}, \mathbf{g}, \mathbf{h})$ Energy profiles at zero bias for the ON and the OFF states of the junctions with $\mathrm{Nb}$ concentrations of 1.0, 0.1 and $0.01 \mathrm{wt} \%$, respectively, where the green and the orange arrows denote the direct tunnelling and the thermally assisted tunnelling, respectively.

dependence upon electric field only at low temperature below $100 \mathrm{~K}$ (ref. 42). Therefore, the $C_{\mathrm{d}}^{-2}-V_{\mathrm{d}}$ plots can be fitted using $C_{\mathrm{d}}^{-2}=2\left(V_{\mathrm{bi}}-V_{\mathrm{d}}\right) / q \varepsilon_{0} \varepsilon_{\mathrm{r}} N_{\mathrm{D}} S^{2}$, which yields the doping concentration $N_{\mathrm{D}}$ in NbSTO from the slope and the build-in potential $V_{\mathrm{bi}}$ of the Schottky barrier from the intercept. $N_{\mathrm{D}}$ values extracted are plotted in Fig. $5 c$, which are comparable to those reported previously in metal/NbSTO Schottky junctions ${ }^{38,43,44}$. $V_{\mathrm{bi}}$ at zero bias are shown in Fig. $5 \mathrm{~d}$. With $V_{\mathrm{bi}}$ and $N_{\mathrm{D}}, W_{\mathrm{d}}$ can be estimated from $W_{\mathrm{d}}=\left(2 \varepsilon_{0} \varepsilon_{\mathrm{r}} V_{\mathrm{bi}} / q N_{\mathrm{D}}\right)^{1 / 2}$, as shown in Fig. $5 \mathrm{e}$. It is obvious that $V_{\mathrm{bi}}$ and $W_{\mathrm{d}}$ in the OFF state is larger than those in the ON state, indicative of the enhanced Schottky barrier in the OFF state and the effective ferroelectric modulation on it.

The energy profile of the Schottky barrier can be deduced in terms of $V_{\mathrm{bi}}$ and $W_{\mathrm{d}}$ obtained above for each $\mathrm{Nb}$ concentration (see Methods section), as shown in Fig. 5f-h. For heavily doped NbSTO with $\mathrm{Nb}$ concentration at 1.0 and $0.7 \mathrm{wt} \%$, the Schottky barrier at the BTO/NbSTO interface is absent in the ON state. Therefore, the transport is governed by direct electron tunnelling through the 4 u.c.-thick BTO barrier (Fig. 5f), as observed in Fig. 3a. Thus, the large $\mathrm{ON}$ state current is observed. When the $\mathrm{Nb}$ concentration decreases to $0.1 \mathrm{wt} \%$, the Schottky barrier appears. This leads to the thermally activated $I-V$ characteristic observed in the $\mathrm{ON}$ state, as shown in Fig. 3c. The direct tunnelling may be suppressed because of the increase in the effective barrier width at $E_{\mathrm{F}}$ with the appearance of the Schottky barrier, which is $3.87 \mathrm{~nm}$ in width and $0.26 \mathrm{eV}$ in height, as extracted from the fitting. However, the thermally assisted tunnelling, in which the electrons are thermally emitted to an energy above $E_{\mathrm{F}}$ and then tunnel through a thinner barrier, is still pronounced with this weak Schottky barrier. This gives the $n$ value greater than unity, as observed in Fig. 4c. The existence of thermally assisted tunnelling currents is confirmed by further analysing temperature-dependent $I-V$ curves (Supplementary Fig. 5) $25,42,45$. Therefore, the FTJ with the Nb concentration at 0.1 wt $\%$ exhibits a large ON state current comparable to the direct tunnelling through the BTO barrier (Fig. 3a). As the $\mathrm{Nb}$ concentration further decreases, both $V_{\mathrm{bi}}$ and $W_{\mathrm{d}}$ increase in the $\mathrm{ON}$ state. The device with the $\mathrm{Nb}$ concentration at $0.01 \mathrm{wt} \%$ exhibits a Schottky barrier of $0.40 \mathrm{eV}$ in height and $168 \mathrm{~nm}$ in width. The thermally assisted tunnelling through the barrier is hence suppressed, in comparison with the transport in the $0.1 \mathrm{wt} \%$ device, and the ON state current decreases, as observed in Fig. 3a,c. 
In the OFF state, the surface of heavily doped NbSTO, with the $\mathrm{Nb}$ concentration at 1.0 and $0.7 \mathrm{wt} \%$, is switched to depletion. For the device with the $\mathrm{Nb}$ concentration at $1.0 \mathrm{wt} \%$, the Schottky barrier is $0.76 \mathrm{eV}$ in height but only $4.02 \mathrm{~nm}$ in width. This thin barrier cannot suppress the thermally assisted tunnelling completely. When the $\mathrm{Nb}$ concentration decreases to $0.1 \mathrm{wt} \%$, the Schottky barrier increases to $1.07 \mathrm{eV}$ in height and $9.68 \mathrm{~nm}$ in width. The OFF state current is then efficiently shut off as the barrier gets high enough and wide enough to suppress the thermally assisted tunnelling. Therefore, a greatly enhanced ON/OFF ratio is achieved at this composition (Fig. 3f). As the $\mathrm{Nb}$ concentration further decreases, $E_{\mathrm{F}}$ drops into the bandgap of NbSTO (see Methods section) and the contact potential is reduced. With $\mathrm{Nb}$ concentration at $0.01 \mathrm{wt} \%, V_{\mathrm{bi}}$ is only $0.64 \mathrm{~V}$, although the barrier extends deep into $\operatorname{NbSTO}\left(W_{\mathrm{d}}=216 \mathrm{~nm}\right)$, as shown in Fig. 5h. With this low Schottky barrier height, the thermally assisted tunnelling becomes pronounced again since the electrons are easier to be emitted to an energy level where the barrier is thin enough to allow the tunnelling. This is in agreement with the observation that the onset of the OFF state current decreases when $\mathrm{Nb}$ concentration decreases from 0.1 to $0.01 \mathrm{wt} \%$. This larger OFF state current results in the smaller ON/OFF ratio observed in this lightly doped composition range, as shown in Fig. 3c.

From the above analyses, one may find that in FTJs with a 4 u.c.-thick BTO barrier, the junction transport is predominantly controlled by the interface Schottky barrier and the BTO ferroelectric barrier itself is negligible to the transport because the ultrathin barrier allows a large tunnelling transmittance, as evidenced in Supplementary Fig. 4. The highest ON/OFF ratio observed at $0.1 \mathrm{wt} \% \mathrm{Nb}$ concentration is a result of the optimized Schottky barrier, which efficiently modulates the thermally assisted tunnelling current between the two resistance states. The optimized FTJ with $0.1 \mathrm{wt} \% \mathrm{Nb}$ concentration exhibits excellent resistance retention with no obvious degradation observed within $12 \mathrm{~h}$ in both the ON and the OFF states, as shown in Fig. 6a. After $2 \times 10^{4}$ bipolar switching cycles, the device still maintains a giant ON/OFF ratio about $1.0 \times 10^{5}$, as shown in Fig. 6b, indicating excellent switching endurance. Data retention and bipolar switching properties of the FTJ with $1.0 \mathrm{wt} \% \mathrm{Nb}$ concentration are shown in Fig. 6 for comparison. In spite of the lower ON/OFF ratio, it also shows good retention and switching endurance.

\section{Discussion}

We have shown that TER in Pt/BTO/NbSTO FTJs can be greatly enhanced by engineering the Schottky barrier. The ON/OFF ratio increases from about 400 to $8 \times 10^{5}$ by simply decreasing the BTO thickness from 16 to 4 u.c., where the OFF state current is significantly suppressed due to the enhancement in the Schottky barrier. The TER is further optimized with varying $\mathrm{Nb}$ concentration. For FTJs with heavily doped NbSTO, the $\mathrm{ON} / \mathrm{OFF}$ ratio is limited by the large OFF state current since the Schottky barrier is too thin to suppress the electron tunnelling. For FTJs with lightly doped NbSTO, the Schottky barrier height is low even though the barrier width is large. This low barrier height facilitates thermally assisted tunnelling in the OFF state and degrades the ON/OFF ratio. With an optimized $\mathrm{Nb}$ concentration at $0.1 \mathrm{wt} \%$, the Schottky barrier is appropriate both in width and in height, which allows a large ON state current but shuts off the current efficiently in the OFF state, giving rise to the highest ON/OFF ratio about $6 \times 10^{6}$.

Recently, Liu et al. ${ }^{46}$ have reported that, in addition to the Schottky barrier, the width of the ferroelectric barrier itself can also be modulated in $\mathrm{SrRuO}_{3} / \mathrm{BTO} / \mathrm{n}-\mathrm{SrTiO}_{3} \mathrm{FTJ}$, where the
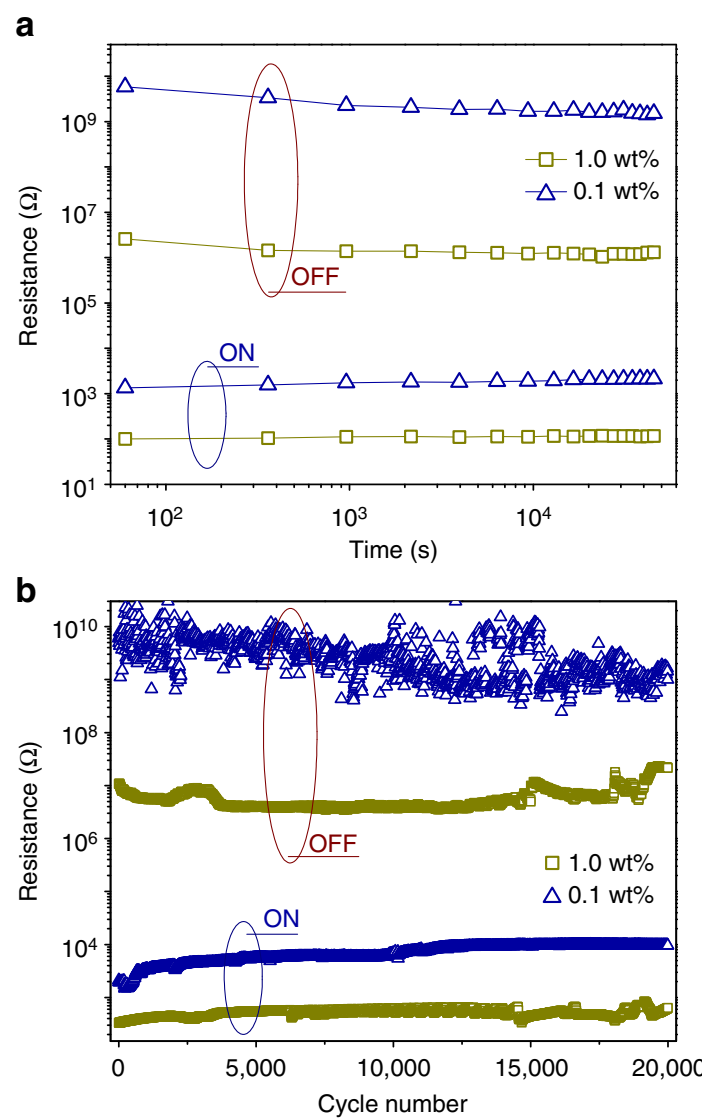

Figure 6 | Room-temperature device endurance of the 4 u.c.-thick $\mathrm{Pt} / \mathrm{BaTiO}_{3} / \mathrm{Nb}: \mathrm{SrTiO}_{3}$ ferroelectric tunnel junctions with $\mathrm{Nb}$ concentrations of $\mathbf{1 . 0}$ and $\mathbf{0 . 1} \mathbf{w t} \%$, respectively. (a) Data retention and (b) bipolar resistance switching. Cycling pulse voltages are $+2.5 /-3.0 \mathrm{~V}$ and $+4.5 /-5.0 \mathrm{~V}$ for the 1.0 and $0.1 \mathrm{wt} \% \mathrm{FTJs}$, respectively. The read pulse voltage is $0.6 \mathrm{~V}$.

BTO surface is metallized in the ON state, due to penetrated electrons from the accumulated n-type $\mathrm{SrTiO}_{3}$ surface. This reversible metallization probably cannot be ruled out in the FTJs with heavily doped NbSTO. However, the largest ON/OFF ratio observed comes from the efficient modulation on thermally assisted tunnelling through a Schottky barrier with an appropriate profile. This highlights the effect of Schottky barrier engineering in performance optimization in MFS-type FTJs. The predominant function of the Schottky barrier is also evidenced by the drastically reduced TER (only about 10, as shown in Supplementary Fig. 6) observed in an Al/BTO/NbSTO (Nb: 0.1 wt\%) FTJ with a 4 u.c.-thick BTO barrier, in which the Schottky barrier is significantly suppressed due to the low work function (about $4.28 \mathrm{eV}$ ) of the Al top electrode.

In summary, the proposed Schottky barrier engineering provides a convenient approach to optimize the TER performance by tuning microstructural parameters. We find that with a 4 u.c.-thick ultrathin BTO barrier, the MFS-type FTJs may be regarded as polarization-modulated metal/semiconductor Schottky junctions, in which an efficient control on the junction transport and a greatly enhanced TER are achieved. Chang and Esaki have proposed a non-volatile Schottky diode based on a MFS tunnelling structure, in which the Schottky barrier height can be modulated by charge/discharge of interfacial states associated with the polarization reversal ${ }^{47}$. In the present work, coherent growth of BTO on lattice-matched NbSTO may greatly 
suppress the interfacial states ${ }^{24,48}$ and the observed TER is from the modulation on the depleted space charge region and hence the Schottky barrier with the polarization reversal. Recently, high-quality $\mathrm{HfO}_{2}$-based ferroelectric ultrathin films have been elaborated directly on $\mathrm{Si}$ substrates ${ }^{49-51}$. With appropriate choice of material parameters, the proposed ferroelectric-modulated Schottky junctions may have potential applications for Si-based memories compatible to the current technology. In addition, the electrically tunable rectifying characteristics and the giant ON/OFF ratio in these devices may help to suppress crosstalk issues in two-terminal crossbar architectures and facilitate the design of high performance memory devices ${ }^{52,53}$.

\section{Methods}

Device preparation. Ultrathin BTO films with thickness of 4-16 u.c. were epitaxially grown on (001) single-crystalline $\mathrm{NbSTO}(\mathrm{Nb}: 0.01-1.0 \mathrm{wt} \%)$ substrates by pulsed laser deposition using a KrF excimer laser (Coherent COMPexPro 201), monitored in situ by reflection high energy electron diffraction. Prior to the deposition, the NbSTO substrates were etched by $\mathrm{NH}_{4} \mathrm{~F}$ buffered-HF solution and then annealed at $950^{\circ} \mathrm{C}$ for $1 \mathrm{~h}$ in flowing $\mathrm{O}_{2}$ to form a $\mathrm{TiO}_{2}$ single-terminated step-terrace surface. The BTO films were deposited with $2.5 \mathrm{~J} \mathrm{~cm}^{-2}$ laser energy density at $2 \mathrm{~Hz}$ repetition, keeping the substrate temperature at $750{ }^{\circ} \mathrm{C}$ and the $\mathrm{O}_{2}$ pressure at $5 \times 10^{-3} \mathrm{mbar}$. Pt top electrodes about $30 \mu \mathrm{m}$ in diameter and $200 \mathrm{~nm}$ in thickness were deposited on the surface of BTO/NbSTO heterostructures by sputtering with a shadow mask to form the FTJs.

Electrical characterizations. The surface morphologies of the BTO/NbSTO heterostructures were recorded by a Park XE-07 AFM. Room-temperature ferroelectric properties of the BTO/NbSTO heterostructures were measured using an Asylum Research Cypher scanning probe microscope with conductive $\mathrm{Pt} / \mathrm{Ti}$-coated tips as the top electrodes. The PFM hysteresis loops were collected in the DART (dual a.c. resonance tracking) mode with triangle pulse waveforms applying on the tip. The piezoresponse $(P R)$ is deduced from the amplitude $(A)$ and the phase $(\theta)$ signals by the formula of $P R=A \cos \theta$. Phase and amplitude images were recorded in the single-frequency PFM mode. Conductive AFM is measured on the Pt electrode of a Pt/BTO/NbSTO FTJ with bias applied on a doped diamond-coated tip (CDT-NCHR, NanoWorld). The resistance switching of the $\mathrm{Pt} / \mathrm{BTO} / \mathrm{NbSTO}$ FTJs were measured by a Keithley 2400 SourceMeter and the low temperature $I-V$ measurements were performed on a LakeShore CRX-4K probe station. $C-V$ characteristics were measured using an Agilent 4294A impedance analyser at room temperature with a frequency of $4 \mathrm{MHz}$ and an oscillation level of $50 \mathrm{mV}$. The testing pulses were applied to the Pt electrodes and the NbSTO substrates were always grounded through indium ohmic contact pads.

Direct tunnelling model. For a trapezoidal potential barrier, the current density $J$ can be described by ${ }^{12}$

$$
\begin{aligned}
J= & C \frac{\exp \left\{\alpha(V)\left[\left(\Phi_{2}-\frac{e V}{2}\right)^{3 / 2}-\left(\Phi_{1}+\frac{e V}{2}\right)^{3 / 2}\right]\right\}}{\alpha^{2}(V)\left[\left(\Phi_{2}-\frac{e V}{2}\right)^{1 / 2}-\left(\Phi_{1}+\frac{e V}{2}\right)^{1 / 2}\right]^{2}} \times \\
& \sinh \left\{\frac{3}{2} \alpha(V)\left[\left(\Phi_{2}-\frac{e V}{2}\right)^{1 / 2}-\left(\Phi_{1}+\frac{e V}{2}\right)^{1 / 2}\right] \frac{e V}{2}\right\}
\end{aligned}
$$

where $C=-\left(4 e m_{\mathrm{n}}^{*}\right) /\left(9 \pi^{2} \hbar^{3}\right)$ and $\alpha(V)=\left[4 d\left(2 m_{\mathrm{n}}^{*}\right)^{1 / 2}\right] /\left[3 \hbar\left(\Phi_{1}+e V-\Phi_{2}\right)\right]$, $\Phi_{1}\left(\Phi_{2}\right)$ is the barrier height at the Pt/BTO (BTO/NbSTO) interface, $m_{\mathrm{n}}^{*}$ the effective electron mass, $\hbar$ the reduced Planck constant, and $d$ the BTO barrier width (approx. $1.6 \mathrm{~nm}$ )

Schottky barrier calculation. Taking $E_{\mathrm{C}}(0)=V_{\mathrm{bi}}$ and $E_{\mathrm{C}}(\infty)=0$ as the boundary conditions, the energy profile $\left[E_{\mathrm{C}}(x)\right]$ for Schottky barrier as a function of distance $(x)$ from the ferroelectric/semiconductor interface can be deduced as follows ${ }^{34}$

$$
E_{\mathrm{C}}(x)=q\left(V_{\mathrm{bi}}+\phi_{\mathrm{n}}\right)-\frac{q^{2} N_{\mathrm{D}}}{\varepsilon_{0} \varepsilon_{\mathrm{r}}}\left(W_{\mathrm{d}} x-\frac{x^{2}}{2}\right)
$$

where $\phi_{\mathrm{n}}$ is the difference between the conduction-band minimum $\left(E_{\mathrm{C}}\right)$ and the $E_{\mathrm{F}}$ of NbSTO, defined as $\left(E_{\mathrm{C}}-E_{\mathrm{F}}\right) / q$, which is negative (positive) for the degenerated (nondegenerated) NbSTO. $\phi_{\mathrm{n}}$ can be simply estimated using the effective mass of electrons $m_{\mathrm{n}}^{*}$ in NbSTO ${ }^{34}$ :

$$
\begin{gathered}
\phi_{\mathrm{n}}=\frac{E_{\mathrm{F}}-E_{\mathrm{C}}}{q} \approx \frac{k_{\mathrm{B}} T}{q}\left[\ln \left(\frac{N_{\mathrm{D}}}{N_{\mathrm{C}}}\right)+2^{-3 / 2}\left(\frac{N_{\mathrm{D}}}{N_{\mathrm{C}}}\right)\right] \\
N_{\mathrm{C}}=2\left(\frac{2 \pi m_{\mathrm{n}}^{*} k_{\mathrm{B}} T}{h^{2}}\right)^{3 / 2}
\end{gathered}
$$

where $N_{\mathrm{C}}$ is the density-of-state of conduction band of NbSTO and $h$ is the Planck constant. The effective Richardson constant of NbSTO in Schottky junctions is, in general, assumed to be $156 \mathrm{~A} \mathrm{~K}^{-2} \mathrm{~cm}^{-2}$, which corresponds to $m_{\mathrm{n}}^{*}=1.3 m_{0}$ $\left(m_{0} \text {, electron mass }\right)^{38,45}$.

Data availability. The data that support the findings of this study are available from the corresponding authors on request.

\section{References}

1. Scott, J. F. Applications of modern ferroelectrics. Science 315, 954-959 (2007)

2. Dawber, M., Rabe, K. M. \& Scott, J. F. Physics of thin-film ferroelectric oxides. Rev. Mod. Phys. 77, 1083 (2005).

3. Fong, D. D. et al. Ferroelectricity in ultrathin perovskite films. Science 304, 1650-1653 (2004).

4. Junquera, J. \& Ghosez, P. Critical thickness for ferroelectricity in perovskite ultrathin films. Nature 422, 506-509 (2003).

5. Kim, Y. S. et al. Critical thickness of ultrathin ferroelectric $\mathrm{BaTiO}_{3}$ films. Appl. Phys. Lett. 86, 102907 (2005).

6. Tsymbal, E. Y. \& Kohlstedt, H. Tunneling across a ferroelectric. Science 313, 181-183 (2006).

7. Garcia, V. \& Bibes, M. Ferroelectric tunnel junctions for information storage and processing. Nat. Commun. 5, 4289 (2014).

8. Zhuravlev, M. Y., Sabirianov, R. F., Jaswal, S. S. \& Tsymbal, E. Y. Giant electroresistance in ferroelectric tunnel junctions. Phys. Rev. Lett. 94, 246802 (2005).

9. Kohlstedt, H., Pertsev, N. A., Contreras, J. R. \& Waser, R. Theoretical currentvoltage characteristics of ferroelectric tunnel junctions. Phys. Rev. B 72, 125341 (2005).

10. Pantel, D. \& Alexe, M. Electroresistance effects in ferroelectric tunnel barriers. Phys. Rev. B 82, 134105 (2010).

11. Garcia, V. et al. Giant tunnel electroresistance for non-destructive readout of ferroelectric states. Nature 460, 81-84 (2009).

12. Gruverman, A. et al. Tunneling electroresistance effect in ferroelectric tunnel junctions at the nanoscale. Nano Lett. 9, 3539-3543 (2009).

13. Chanthbouala, A. et al. Solid-state memories based on ferroelectric tunnel junctions. Nat. Nanotech. 7, 101-104 (2012).

14. Pantel, D., Goetze, S., Hesse, D. \& Alexe, M. Reversible electrical switching of spin polarization in multiferroic tunnel junctions. Nat. Mater. 11, 289-293 (2012).

15. Garcia, V. et al. Ferroelectric control of spin polarization. Science 327, 1106-1110 (2010).

16. Wen, Z., You, L., Wang, J., Li, A. \& Wu, D. Temperature-dependent tunneling electroresistance in $\mathrm{Pt} / \mathrm{BaTiO}_{3} / \mathrm{SrRuO}_{3}$ ferroelectric tunnel junctions. Appl. Phys. Lett. 103, 132913 (2013).

17. Zenkevich, A. et al. Electronic band alignment and electron transport in $\mathrm{Cr} / \mathrm{BaTiO}_{3} / \mathrm{Pt}$ ferroelectric tunnel junctions. Appl. Phys. Lett. 102, 062907 (2013).

18. Hambe, M. et al. Crossing an interface: ferroelectric control of tunnel currents in magnetic complex oxide heterostructures. Adv. Func. Mater. 20, 2436-2441 (2010).

19. Bruno, F. Y. et al. Millionfold resistance change in ferroelectric tunnel junctions based on nickelate electrodes. Adv. Electro. Mater. 2, 1500245 (2016).

20. Kim, D. J. et al. Ferroelectric tunnel memristor. Nano Lett. 12, 5697-5702 (2012).

21. Wang, L. et al. Overcoming the fundamental barrier thickness limits of ferroelectric tunnel junctions through $\mathrm{BaTiO}_{3} / \mathrm{SrTiO}_{3}$ composite barriers. Nano Lett. 16, 3911-3918 (2016).

22. Zhuravlev, M. Y., Wang, Y., Maekawa, S. \& Tsymbal, E. Y. Tunneling electroresistance in ferroelectric tunnel junctions with a composite barrier. Appl. Phys. Lett. 95, 052902 (2009).

23. Yin, Y. W. et al. Enhanced tunneling electroresistance effect due to a ferroelectrically induced phase transition at a magnetic complex oxide interface. Nat. Mater. 12, 397-402 (2013).

24. Wen, Z., Li, C., Wu, D., Li, A. \& Ming, N. Ferroelectric-field-effect-enhanced electroresistance in metal/ferroelectric/semiconductor tunnel junctions. Nat. Mater. 12, 617-621 (2013)

25. Hu, W. J., Wang, Z., Yu, W. \& Wu, T. Optically controlled electroresistance and electrically controlled photovoltage in ferroelectric tunnel junctions. Nat. Commun. 7, 10808 (2016).

26. Hwang, C. S. Prospective of semiconductor memory devices: from memory system to materials. Adv. Electron. Mater. 1, 1400056 (2015).

27. Park, J. H. et al. A hybrid ferroelectric-flash memory cells. J. Appl. Phys. 116, 124512 (2014).

28. Bang, T. et al. Low-frequency noise characteristics in SONOS flash memory with vertically stacked nanowire FETs. IEEE Electron. Device Lett. 38, 40-43 (2017). 
29. Kwon, W., Park, I. J. \& Shin, C. Highly scalable NAND flash memory cell design embracing backside charge storage. J. Semicond. Tech. Sci. 15, 286-291 (2015).

30. Jesse, S., Baddorf, A. P. \& Kalinin, S. V. Switching spectroscopy piezoresponse force microscopy of ferroelectric materials. Appl. Phys. Lett. 88, 062908 (2006).

31. Balke, N. et al. Differentiating ferroelectric and nonferroelectric electromechanical effects with scanning probe microscopy. ACS Nano 9, 6484-6492 (2015).

32. Bark, C. W. et al. Switchable induced polarization in $\mathrm{LaAlO}_{3} / \mathrm{SrTiO}_{3}$ heterostructures. Nano Lett. 12, 1765-1771 (2012).

33. Strelcov, E. et al. Role of measurement voltage on hysteresis loop shape in Piezoresponse Force Microscopy. Appl. Phys. Lett. 101, 192902 (2012).

34. Sze, S. M. \& Ng, K. K. in Physics of Semiconductor Devices 3rd edn (Wiley, 2007).

35. Yoshida, Y., Tamura, H., Gotoh, K., Takauchi, H. \& Hasuo, S. Electrical properties of $\mathrm{Au} /$ and $\mathrm{YBa}_{2} \mathrm{Cu}_{3} \mathrm{O}_{7-\mathrm{x}} / \mathrm{SrTi}_{1-\mathrm{y}} \mathrm{Nb}_{\mathrm{y}} \mathrm{O}_{3}$ diodes. J. Appl. Phys. 70, 4976 (1991)

36. Gao, X. S., Liu, J. M., Au, K. \& Dai, J. Y. Nanoscale ferroelectric tunnel junctions based on ultrathin $\mathrm{BaTiO}_{3}$ film and Ag nanoelectrodes. Appl. Phys. Lett. 101, 142905 (2012).

37. Chu, J. L., Persky, G. \& Sze, S. M. Thermionic injection and space-chargelimited current in reach-through $\mathrm{p}^{+} \mathrm{np}^{+}$structures. J. Appl. Phys. 43, 3510 (1972).

38. Suzuki, S. et al. Fabrication and characterization of $\mathrm{Ba}_{1-\mathrm{x}} \mathrm{K}_{\mathrm{x}} \mathrm{BiO}_{3} / \mathrm{Nb}$-doped $\mathrm{SrTiO}_{3}$ all-oxide-type Schottky junctions. J. Appl. Phys. 81, 6830 (1997).

39. Mikheev, E., Hoskins, B. D., Strukov, D. B. \& Stemmer, S. Resistive switching and its suppression in $\mathrm{Pt} / \mathrm{Nb}: \mathrm{SrTiO}_{3}$ junctions. Nat. Commun. 5, 3990 (2014).

40. Card, H. C. \& Rhoderick, E. H. Studies of tunnel MOS diodes I. Interface effects in silicon Schottky diodes. J. Phys. D: Appl. Phys. 4, 1589 (1971)

41. Barrett, J. H. Dielectric constant in perovskite type crystals. Phys. Rev. 86, 118 (1951).

42. Susaki, T., Kozuka, Y., Tateyama, Y. \& Hwang, H. Y. Temperature-dependent polarity reversal in Au/Nb:SrTiO 3 Schottky junctions. Phys. Rev. B 76, 155110 (2007).

43. Hikita, Y., Kawamura, M., Bell, C. \& Hwang, H. Y. Electric field penetration in $\mathrm{Au} / \mathrm{Nb}: \mathrm{SrTiO}_{3}$ Schottky junctions probed by bias-dependent internal photoemission. Appl. Phys. Lett. 98, 192103 (2011).

44. Spinelli, A., Torija, M. A., Liu, C., Jan, C. \& Leighton, C. Electronic transport in doped $\mathrm{SrTiO}_{3}$ : conduction mechanisms and potential applications. Phys. Rev. B 81, 155110 (2010).

45. Cuellar, F. A. et al. Thermally assisted tunneling transport in $\mathrm{La}_{0.7} \mathrm{Ca}_{0.3} \mathrm{MnO}_{3} /$ $\mathrm{SrTiO}_{3}: \mathrm{Nb}$ Schottky-like heterojunctions. Phys. Rev. B 85, 245122 (2012).

46. Liu, X., Burton, J. D. \& Tsymbal, E. Y. Enhanced tunneling electroresistance in ferroelectric tunnel junctions due to the reversible metallization of the barrier. Phys. Rev. Lett. 116, 197602 (2016).

47. Chang, L. L. \& Esaki, L. Nonvolatile Schottky diode with barrier height controlled by ferroelectric polarization. IBM Tech. Discl. Bull. 14, 1250 (1971).

48. Mathews, S., Ramesh, R., Venkatesan, T. \& Benedetto, J. Ferroelectric field effect transistor based on epitaxial perovskite heterostructures. Science 276, 238-240 (1997).
49. Chernikova, A. et al. Ultrathin $\mathrm{Hf}_{0.5} \mathrm{Zr}_{0.5} \mathrm{O}_{2}$ ferroelectric films on Si. ACS Appl. Mater. Interfaces 8, 7232-7237 (2016).

50. Müller, J. et al. Ferroelectric hafnium oxide: a CMOS-compatible and highly scalable approach to future ferroelectric memories http://dx.doi.org/10.1109/ IEDM.2013.6724605 (2013).

51. Park, M. H. et al. Ferroelectricity and antiferroelectricity of doped thin $\mathrm{HfO}_{2}$-based films. Adv. Mater. 27, 1811-1831 (2015).

52. Linn, E., Rosezin, R., Kügeler, C. \& Waser, R. Complementary resistive switches for passive nanocrossbar memories. Nat. Mater. 9, 403-406 (2010).

53. Lee, M. et al. A fast, high-endurance and scalable non-volatile memory device made from asymmetric $\mathrm{Ta}_{2} \mathrm{O}_{5-\mathrm{x}} / \mathrm{TaO}_{2-\mathrm{x}}$ bilayer structures. Nat. Mater. 10, 625-630 (2011).

\section{Acknowledgements}

This work was jointly sponsored by State Key Program for Basic Research of China (2015CB921203) and Natural Science Foundation of China (11574169, 51302139, 11374139). Z.W. acknowledges financial support from the Hong Kong Scholars Program (XJ2015024).

\section{Author contributions}

Z.W. and D.W. conceived this work and designed the experiment. Z.X. and C.Z. fabricated the devices. Z.X. performed the electrical measurements. Z.W., C.L. and Z.X performed the SPM measurements. Z.W., D.W., Z.X., J.R., C.L., A.L. and J.D. analysed the data. Z.W. and D.W. wrote the manuscript. All authors discussed the data and contributed to the manuscript.

\section{Additional information}

Supplementary Information accompanies this paper at http://www.nature.com/ naturecommunications

Competing interests: The authors declare no competing financial interests.

Reprints and permission information is available online at http://npg.nature.com/ reprintsandpermissions/

How to cite this article: $\mathrm{Xi}, \mathrm{Z}$. et al. Giant tunnelling electroresistance in metal/ferroelectric/semiconductor tunnel junctions by engineering the Schottky barrier. Nat. Commun. 8, 15217 doi: 10.1038/ncomms15217 (2017).

Publisher's note: Springer Nature remains neutral with regard to jurisdictional claims in published maps and institutional affiliations.

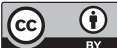

This work is licensed under a Creative Commons Attribution 4.0 International License. The images or other third party material in this article are included in the article's Creative Commons license, unless indicated otherwise in the credit line; if the material is not included under the Creative Commons license, users will need to obtain permission from the license holder to reproduce the material. To view a copy of this license, visit http://creativecommons.org/licenses/by/4.0/

(C) The Author(s) 2017 